\title{
Challenge of Attaining Flow Arrest in Anterior Circulation Tandem Occlusions in Large Vessel Ischemic Stroke: Wedged SAVE Technique
}

\author{
Volker Maus $^{\mathrm{a}} \quad$ Utako Birgit Barnikol $^{\mathrm{b}} \quad$ Anastasios Mpotsaris $^{\mathrm{c}}$ \\ ${ }^{a}$ Department of Neuroradiology, University Medical Center Göttingen, Göttingen, Germany; ${ }^{\mathrm{b}}$ Department of \\ Child and Adolescence Psychiatry, Research Unit Ethics and University Hospital Cologne, Cologne, Germany; \\ 'Department of Neuroradiology, University Hospital of Aachen, Aachen, Germany
}

In his letter, Zhao [1] mentions that in patients with severe stenosis, an aspiration catheter might be unable to pass through the lesion and that this might lead to adopting an antegrade treatment strategy. In these patients, one meaningful strategy is to first carefully dilate the proximal lesion by using balloon angioplasty to enable the aspiration catheter/guide catheter to pass through easily for performing thrombectomy; subsequent carotid artery stenting can then be performed. Thus, the use of balloon angioplasty was chosen for our propensity matched analysis in order to adjust for this possible confounder.

We agree that the use of a balloon guide catheter might have an influence on the reperfusion success. However, the use of a balloon guide catheter was mentioned in the Method section and applied in both groups. As described, in a few patients with retrograde treatment, proxi- mal flow arrest could even be achieved when passing the extracranial lesion with a non-balloon guide catheter or aspiration catheter, which occluded the internal carotid artery (ICA) and led to proximal flow control. Furthermore, the so-called Stent-retriever Assisted Vacuum-locked Extraction (SAVE) technique was used in part in both groups, and this might contribute to proximal flow control (arrest or reversal) by aspiration through the guider $[2,3]$. Such an example of retrograde treatment by applying the SAVE technique in a case with extracranial pseudoocclusion of the ICA and intracranial Carotid-T-occlusion is illustrated: First the extracranial stenosis is depicted (Fig. 1a), then by a roadmap, the microcatheter is negotiated to the M1-Segment of the MCA, placing a long stent-retriever (Trevo ProVue $4 \times 40 \mathrm{~mm}$ ) as previously described $[2,3]$. This acts then as "anchor" for the navigation of the aspiration catheter, in this case a Catalyst 5 (Stryker Neurovascular, Kalamazoo, MI, USA) for facilitating the passing of the carotid pseudo-occlusion. This results in a "wedged SAVE" (Fig. 1b), as the aspiration catheter occludes the extracranial ICA, leading to flow-arrest. The maneuver is then conducted by retrieving the stent-retriever and aspiration catheter as entity into the guide catheter placed proximally to the pseudo-occlusion, leading to a near-complete TICI $2 c$ reperfusion result (Fig. 1c) with a "widened aspect" of the initial extracranial pseudo-occlusion (Fig. 1d). Postinterventional MRI at 5 days demonstrates the safety of the maneuver (Fig. 1e-g); only 3 microemboli that might be related to the procedure are depicted in addition to the underlying basal ganglia infarction after successful revascularization of the Carotid-T.

\section{KARGER}

(C) 2018 S. Karger AG, Basel

E-Mail karger@karger.com

www.karger.com/ced
Dr. Volker Maus

Universitätsmedizin Göttingen

Neuroradiologie, Robert-Koch-Strasse 40

DE-37075 Göttingen (Germany)

E-Mail volker.maus@med.uni-goettingen.de 

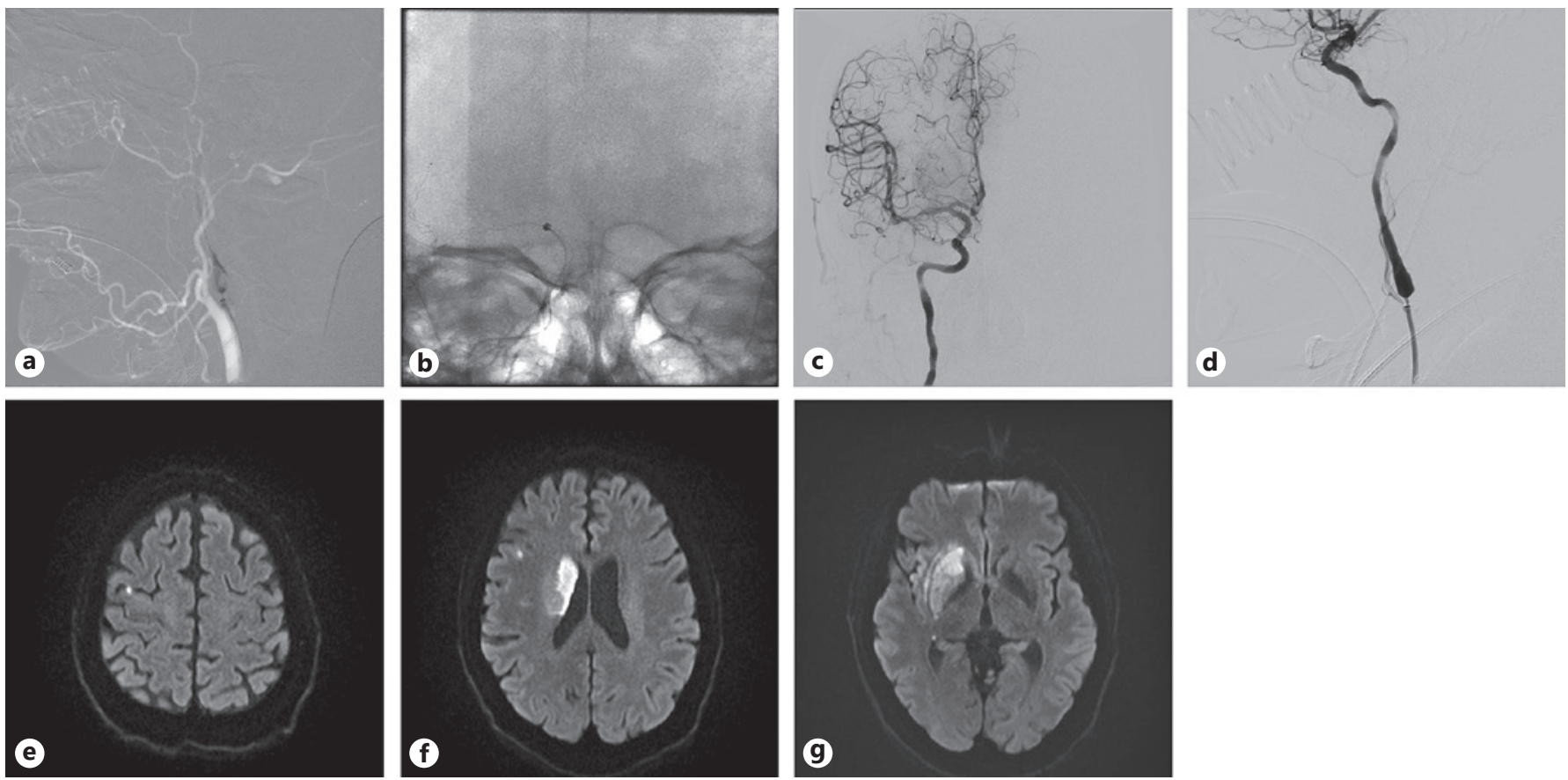

Fig. 1. a Stenosis of the internal carotid artery in a patient with extracranial pseudo-occlusion of the ICA and intracranial carotidT-occlusion. b Distal positioning of a long stent-retriever (Trevo ProVue $4 \times 40 \mathrm{~mm}$ ) in the M1 segment with subsequent navigation of the aspiration catheter. This results in a "wedged SAVE", as the aspiration catheter occludes the extracranial ICA, leading to flow-

arrest. c After one retrieval maneuver, the intracranial occlusion is successfully recanalized with near-complete reperfusion. d "Widened aspect" of the initial extracranial pseudo-occlusion before carotid stenting. e-g Postinterventional MRI at 5 days with only 3 microemboli, which might be related to the procedure.

\section{References}

1 Zhao H: Letter to the article by Maus et al.: Order of treatment matters in ischemic stroke: mechanical thrombectomy first, then carotid artery stenting for tandem lesions of the anterior circulation. Cerebrovasc Dis 2018;46:210.
2 Maus V, Behme D, Kabbasch C, Borggrefe J, Tsogkas I, Nikoubashman O, Wiesmann M, Knauth M, Mpotsaris A, Psychogios MN: Maximizing first-pass complete reperfusion with SAVE. Clin Neuroradiol 2018;28:327338.
3 Maus V, Henkel S, Riabikin A, Riedel C, Behme D, Tsogkas I, Hesse AC, Abdullayev $\mathrm{N}$, Jansen O, Wiesmann M, Mpotsaris A, Psychogios MN: The SAVE technique : largescale experience for treatment of intracranial large vessel occlusions. Clin Neuroradiol 2018, Epub ahead of print. 